\title{
Les études théoriques et expérimentales pour la construction des ouvrages hydrauliques
}

\section{Theoretical and experimental studies for construction of hydraulic structures}

\author{
Exposí De M. NTZTERY \\ INCHNIEUR EN CHEF DES PONTS ET CHAUSSÉLS,

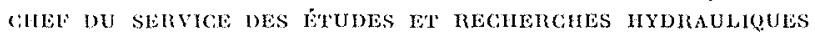 \\ J'ĽLEGTRICITÉ DE FRANCE
}

Monsieur le Président,

Madame,

Messieurs,

Tout le monde est à peu près d'accord maintenant pour admettre que le travail de recherche est devenu inaccessible à l'individu isolé, mais qu'il doit être mené en équipe. Mais la plupart des gens pensent que la nécessité du travail en équipe est due à l'ampleur des moyens expérimentaux à mettre en ouve et qui echappent atux possibilités de l'individu. On évoque toul naturellement les grandes organisations de physiciens et de techniciens qui, quasiment enfermés dans une forteresse, ont pensé et réalisé les premières piles atomiques.

Je voudrais vous montrer sur un exemple concret intéressant les travaux hydrauliques que ce travail d'équipe est quelque chose de beaucoup plus complexe qu'on ne le pense d'ordinaire et que l'équipe peut être souvent une véritable armée, mais dont le défaut principal est la dispersion.

J'ai choisi, à cet efret, le problème du débit solide.

Comme vous le savez, il s'agit là des phénomines d'entraînement de matériaux solides par les cours d'eau, ou d'une manière plus générale par l'eau en mouvement. Ce problème joue un rôle considérable dans l'art de l'ingénieur, qu'il s'agisse de la construction de prises d'eau d'usines hydro-électriques, d'aménagement de cour's d'eau pour la navigation, de la défense des terres cultivables contre l'érosion, de la défense des rivages de la mer contre l'action de la houle et des courants, ou de l'aménagement des ports en plage de sable... et j'en passe.

Comment ce problème a-t-il été attaqué? Bien sùr, des descriptions qualitatives ont été données de tous temps de ses effets les plus spectaculaires et les constructeurs n'ont pas attendu te laboratoire pour tenter de remédier à ces effets. Mais les premières études systématiques dans ee domaine ont été faites par l'ingénieur français Du Boys qui jeta vers 1880 les premières bases d'une théoric du phénomène de charriage en vue des travaux d'aménagement du Rhône. Plus tard, vers 1914, le séologue américain Gilbert attaqua le probleme par la voie expérimentale dans le but de comprendre d'une manière quantitative les phénomènes d'érosion. Vous voyez déjà apparaîttre, dans l'esprit des. chercheurs, des buts bien différents : le but concret d'un aménagement pour l'un, la connaissance générale des phénomènes naturelș pour l'autre. Le développement de l'étude du débit solide s'est poursuivi depuis dans ce double esprit et il a fait appel à des catégories d'individus de plus en plus étendues.

C'est ainsi que les théoriciens de la mécanique de fluides se sont mis de la partie, en cherchant à expliquer les transports de matériaux en suspension, par le mécanisme de 
l'écoulement turbulent (Schmidt, Richardson, puis Rouse, O'Brien, Vanoni). Pendant ce temps, les expérimentateurs et les statisticiens (White, Indri, Mryer-Peter, Ennstein fils) cherchaient à tâtons les lois du charriage.

Parallèlement à ces efforts, dans la voie scientifique, les ingénieurs « du tas », ceux qui sont en prise directe avec les difficultés de réalisation, observaient dans le réel les méfaits du débit solide; les ingénieurs des Ponts et Chaussées s'efforçaient de corriger des lits de rivières vagabondes, les constructeurs d'usines hydroélectriques amélioraient la conception des prises d'eau en rivière. Les ingénieurs maritimes canalisaient cette force à leur profit en façonnant les plages, où le sable défile, à l'aide d'épis d'abord mal placés puis, l'expérience aidant, de plus en plus efficaces; les forestiers cherchaient à fixer leurs torrents en luttant contre le débit solide près de sa source, c'est-à-dire dans la partie haute des bassins versants. Les agriculteurs se sont mis de la partie depuis quelques années et voient maintenant dans ces matériaux arrachés, à la fois l'ennemi qui épuise les terres et l'ami qui en fertilisera peut-être d'autres. La création du Soil Conservation américain et le rôle important qu'y joue l'étude du débit solide, en cst le témoignage le plus important.

Je n'aurai garde d'oublier naturellement dans cette revue ceux qui, professionnellement sont attachés à cette tâche, les hydrauliciens de laboratoire et les hydrologues et hydrauliciens de lerrains.

Ainsi l'équipe dont nous parlions plus haut est devenue une immense armée. On y trouve, comme dans les sports, des amateurs et des professionnels, on y trouve des gens de formations complétement différentes, presque opposées, des physiciens et des naturalistes, des chercheurs purs et des praticiens dont le but concret doit être atteint tout de suite et pas demain.

Dans ce fouillis apparent, deux tendances bien nettes : ceux qui cherchent à comnaître en espérant qu'un jour cela servira à quelque chose, ceux qui cherchent à résoudre un problème parliculier et que la nécessité amène à remonter vers la connaissance générale.

Devant cette diversité quelle peut être l'attitude du responsable des denicrs (publics ou privés). Il sera tenté surtout par l'étude des cas particuliers dont la rentabilité lui apparaîtra plus facilement. Par exemple, on a à construire un canal dérivant les eaux d'une rivière aux caux chargées de limons, vers une usine qui les restituera après utilisation dans un bassin fréquenté par la navigation. Les matériaux en suspension iraient se déposer dans les bassins où ils devraient être dragués. Pour éviter cet inconvénient, on prévoit un bassin de décantation en tête de la dérivation. Sans bassin il faudrait draguer tous les limons; avec un bassin de dicantation ultra perfectionné, les dragages seront réduits à zéro. Mais le coût de la décantation au mètre cube décanté sera plus élevé que le coût des dragages. Il y aura donc un optimum à chercher, ce qui permettra de chifrer la rentabilité de l'étude.

Un autre cas typique se présente lorsque l'étude permet de réduire le coût de l'ouvage. C'est ce que l'on rencontre fréquemment dans la construction des ouvrages hydro-électriques. Ainsi par l'amélioration des formes d'entrée, on a pu réecmment réduire de $30 \%$ la surface des vannages d'un gros ouvrage de déversement. La rentabilité est facile à chiffrer. L'étude paye.

Dans d'autres cas, la rentabilité sera moins facile à chiffrer, mais quand même estimable. Elle pourra prendre le caractère d'une prime d'assurance quand la sécurité de l'ouvrage ou de la région est en jeu. Mais le terrain reste solide et l'on n'hésitera pas à engager des dépenses dont le rapport est immédiat et palpable.

Supposons maintenant un expérimentateur ou un théoricien qui se propose d'entreprendre une étude générale, par exemple l'étude du mécanisme de la suspension ou un système nouveau dont on attend des merveilles pour décanter les eaux. Le financier sera peut-ètre attiré par les applications multiples, même lointaines, de ces recherches. Mais il aura peur de l'échec. Et c'est bien là le noud de la question. Toute recherche d'une solution nouvelle d'un problème scientifique ou technique comporte par nature un risque. Une trouvaille se paye nécessairement par des échecs.

Dans ce domaine, il faudra donc qu'il joue. Mais on sent bien quand même que, dans ce jeu, il faut mettre toutes les chances de son côté et réduire les risques d'échee au minimum. Le moyen le plus efficace qui s'ofre à cet égard, c'est la confrontation permanente des idées et des résultats de toute cette cohorte dispersée que je vous ai montrée tout à l'heure, dans l'exemple que j'ai pris, attelée à la même tâche. Cette confrontation peut naturellement se faire par les relations personnelles. Mais les relations personnelles ne suffisent pas. Il faut la publication, la discussion publique. On obtient ainsi une certaine sélection des idées, qui permet de faciliter au responsable la tâche difficile de placer ses chances dans les meilleurs conditions.

Cette confrontation qui associe, comme nous le voyons souvent aux séances de notre Société, de grands esprits et de jeunes chercheurs passionnés, des hommes de laboratoire et des hommes d'action, des spécialistes d'horizons opposés, a au surplus l'avantage inestimablo d'ouvrir aux uns et aux autres des idées qui leur étaient étrangères. 
J'évoquerai ici une étude qui est maintenant à l'ordre du jour dans un grand nombre de pays, celle du dévasement des grandes retenues hydrauliques. Vous savez que la propagation des eaux turbides dans les grandes masses d'eau des lacs artificiels se présente sous la forme de courants de densité, les couches d'eau turbides des cours d'eau débouchant dans le lac se mélangeant difficilement aux eaux claires de celui-ci. Eh bien! Savez-vous que le problème a été abordé en France pour la première fois par le Laboratoire Dauphinois d'Hydraulique à l'oceasion d'un travail bien différent: l'énergie thermique des mers. L'histoire a commencé en 1942. Notre souci était alors de connaitre l'écoulement des couches d'eaux inégalement salées au voisinage de notre prise d'eau froide sous-marine. Là aussi, il s'agissait de courants de densité. L'expérience et la théorie qui nous ont permis de résoudre notre problème du moment ont ouvert la voie et l'avance prise dans ce domaine nous est maintenant précieuse pour l'étude des dévasements de retenue.

Mais, qui mieux est, ces courants de densité, voici les physiciens de l'atmosphère qui les retrouvent dans la marche de leurs nuages. Ce ne sont plus des eaux salées, ni des eaux turbides, mais des couches d'air plus ou moins chaudes qui hésitent à se mélanger et qui provoquent cette curieuse stratification de l'atmosphère à laquelle on assiste lorsque, le soir tombant, le gradient des températures s'inverse près du sol.

Ainsi voilà un sujet a priori bien spécial qui se retrouve dans plusieurs domaines bien éloignés les uns des autres. La condition essentielle du progrès est donc la rencontre des spécialistes de disciplines diverses. Les uns et les autres y puisent des idées et profitent de l'expérience commune.

Nous avons assisté il y a quelque temps à une série de discussions organisées par notre Société sous la présidence de M. I'Ingénieur HupNer sur les méthodes d'évaluation et de prévision des crues. Cette question capitale sur la conception des ouvrages hydro-électriques et pour l'aménagement des cours d'eau est extrèmement controversée et elle est traitée par des méthodes très nombreuses, toutes naturellement aussi bonnes les unes que les autres. Ce qui aggrave le cas, c'est que, de même que pour notre débit solide, des quantités de gens s'en occupent avec des buts et des processus de pensée bien différents. Géographes, hydrologues, météorologistes, mathématiciens (probabilistes et statisticiens) et puis aussi naturellement ingénieurs, ceux qui en définitive auront la responsabilité des choix du débit.

A ces réunions, toutes ces tendances étaient largement représentées. Le cœur même du problème a été abordé et la valeur de nos extrapolations a été discutée à fond. J'ai, pour ma part, la conviction que ce travail a été très fructueux car, je dois le dire, grâce à un excellent président et à un rapporteur d'une grande clarté, il a été possible de dégager des orientations nouvelles vers l'utilisation combinée de la climatologie et de la statistique à un problème que d'aucuns pouvaient penser aboutir à une impasse.

C'est sur cet exemple que je terminerai car il me paraît être la meilleure preuve - la preuve par les actes - de l'efficacité de nos confrontations d'idées.

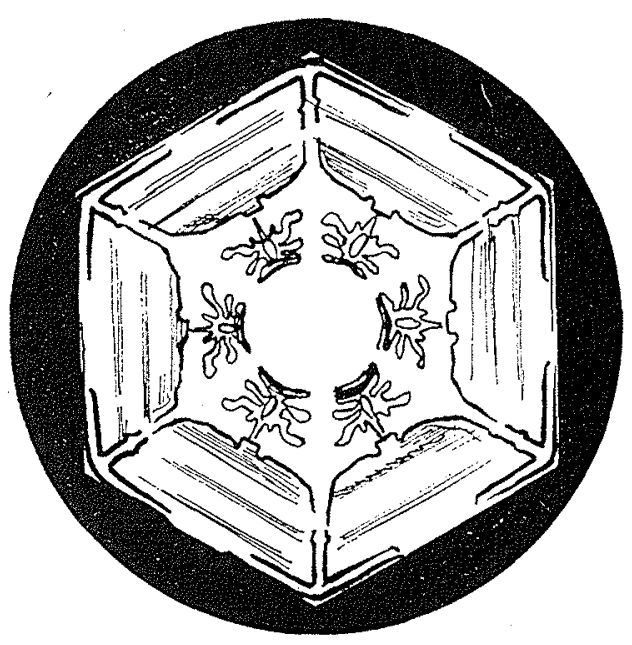

